\title{
INTERACTION OF RIM SEAL AND ANNULUS FLOWS IN AN AXIAL FLOW TURBINE
}

\author{
C. Cao and J. W. Chew \\ Fluids Research Centre \\ School of Engineering \\ University of Surrey \\ Guildford, Surrey, GU2 7XH, UK
}

\author{
P.R. Millington and S.I. Hogg \\ Alstom Power \\ Newbold Road \\ Rugby \\ Warwickshire, CV21 3NH, UK
}

\begin{abstract}
A combined computational fluid dynamics (CFD) and experimental study of interaction of main gas path and rim sealing flow is reported. The experiments were conducted on a two stage axial turbine and included pressure measurements for the cavity formed between the stage 2 rotor disc and the upstream diaphragm for two values of the diaphragm-to-rotor axial clearance. The pressure measurements indicate that ingestion of the highly swirling annulus flow leads to increased vortex strength within the cavity. This effect is particularly strong for the larger axial clearance. Results from a number of steady and unsteady CFD models have been compared to the measured results. Good agreement between measurement and calculation for time-averaged pressures was obtained using unsteady CFD models, which predicted previously unknown unsteady flow features. This led to fast response pressure transducer measurements being made on the rig, and these confirmed the CFD prediction.
\end{abstract}

\section{NOMENCLATURE}

$\mathrm{C}_{\mathrm{o}} \quad$ Stage heat drop expressed as a velocity $\left(\mathrm{C}_{\mathrm{o}}{ }^{2} / 2=\right.$ stage specific heat drop $)$

$\mathrm{C}_{\mathrm{p}}$ pressure coefficient $\left(=2\left(\mathrm{p}-\mathrm{p}_{\mathrm{o}}\right) /\left(\rho \mathrm{C}_{\mathrm{in}}^{2}\right)\right)$

$\mathrm{C}_{\mathrm{w}}$ rim seal mass flow coefficient $\left(=\mathrm{m} / \mu \mathrm{r}_{\mathrm{o}}\right)$

$\mathrm{C}_{\text {in }}$ inlet velocity

$\mathrm{m}$ rim seal mass flow

p pressure 
$\mathrm{p}_{\mathrm{o}}$ static pressure at outer radius of disc

$\operatorname{Re}_{\phi}$ rotational Reynolds number $\left(=\rho \Omega \mathrm{r}_{\mathrm{o}}{ }^{2} / \mu\right)$

u moving blade root speed

$r_{o} \quad$ outer radius of disc

$\mu \quad$ viscosity

$\rho$ density

$\Omega \quad$ angular velocity of rotor

\section{INTRODUCTION}

The present study considers interaction of rim sealing and main annulus flows in an axial flow turbine. In any such turbine with shrouded blades and vanes there will be gaps between rotating and stationary sections of the annulus walls. These gaps must accommodate relative movements of the rotating and stationary components, and will allow flow exchange between the main annulus and the cavity between the rotating and stationary discs. To avoid overheating of turbine discs it is sometimes necessary to channel cool, high pressure air from the compressor to the gap in the turbine inner annulus wall where it is ejected back into the main gas path. In this case the degree of hot gas ingestion, the level of sealing flow required, and the spoiling effects of the sealing flow in the turbine are all of interest. In lower pressure turbine stages ingestion of hot gas may not be of such concern, although the influence of flow through the gap on turbine performance is still of interest. A variety of disc cavity and rim seal geometries are found in current gas and steam turbines, and the influence of geometry on sealing and performance is of considerable interest.

Previous work on turbine rim sealing has noted both the effects of the rotating disc, which tends to draw flow in through the seal (e.g. Bayley and Owen [1]), and the importance of circumferential pressure gradients in the annulus, which can promote inflow and outflow around the seal circumference (e.g. Campbell [2]). It is now generally thought that, at engine conditions, circumferential pressure asymmetries are the primary cause of ingestion, with asymmetries from both stationary vanes and rotating blades contributing. The combined effects of rotating and stationary pressure asymmetries have been considered recently by Bohn et al [3], Roy et al [4], Hills et al [5], and Gentilhomme et al [6]. It may be concluded that predictive methods for rim seal ingestion must take account of the unsteady nature of the flow. Roy et al measured pressures and ingestion in a turbine rig. Based on their unsteady pressure measurements and the observation that steady CFD models did not correctly predict ingestion they concluded that the unsteadiness was playing an important part in the ingestion process. Hills et al showed that an unsteady CFD model gave considerably better agreement 
with previously published experimental ingestion data than steady models. For the conditions considered it was shown that the circumferential pressure asymmetry due to rotating blades had a large influence on ingestion despite being considerably smaller than the pressure asymmetry due to the stationary vanes. This was explained by considering inertial effects on the flow and the fact that the swirl velocity of the air was much closer to the rotor speed than to that of the vanes. An elementary model, taking some account of these effects was also shown to capture the experimental trends.

As noted by Smout et al [7], the experimental rig described by Bohn et al [8] has been used for research under the European collaborative ICAS-GT research program. At some conditions measurements indicated the presence of large scale, low frequency pressure fluctuations with a characteristic wave length greater than that associated with the pitch of the blade or vanes. This result is very relevant to the present study. A further relevant and very recent result is that of Autef [9], as described by Chew et al [10]. Autef found that unsteady CFD calculations for a rotor/stator disc cavity with axisymmetric, steady boundary conditions show 3D unsteady flow at the rim seal for the case of weak external flow. This unsteady flow feature had not been identified previously and calculated levels of ingestion (due to disc pumping) showed much better agreement with experiment than earlier steady CFD models.

In this paper a combined CFD and experimental study of interaction of main gas path and rim sealing flow is reported. Emphasis is on the unsteady nature of the flow and ingestion into the disc cavity. The experiments were conducted on the two stage axial turbine described in the next section, and the FLUENT CFD code was used as described in section 3. Results are presented and compared in section 4, with the main conclusions being summarized in section 5 .

\section{EXPERIMENTAL RIG AND TESTS}

The experimental data were obtained using the ALSTOM two stage HP/IP model air turbine situated in Rugby UK. The facility is primarily used to develop both impulse and reaction steam turbine blading and to provide high quality test data for CFD code validation.

A typical turbine configuration is shown in fig. 1. The turbine incorporates a split shaft arrangement, each shaft having a torque meter, for accurate measurement of shaft power, and a hydraulic dynamometer to absorb the power. Shaft bearing losses are measured separately using a 'balanced bearing system'.

The present tests were carried out using ALSTOM standard impulse HP/IP blading. The first stage of the turbine is used to provide representative flow conditions into the second stage. Interest is focused on interaction of sealing and main annulus flow at the disc rim 
gap, which is shown in fig. 2. The second stage has 50 fixed vanes and 67 rotating blades. The moving blade root diameter is 609.6 $\mathrm{mm}$. Balance holes in the rotor discs are provided to swallow most of the flow from the fixed blade diaphragm gland, with the remaining leakage flow flowing radially outwards into the annulus. The standard disc to stator/diaphragm gap is $4 \mathrm{~mm}$. In some tests this was reduced to $2 \mathrm{~mm}$ by gluing a spacer to the diaphragm face, with finishing to remove any step on the blade root and wall. The spacer extended $15 \mathrm{~mm}$ radially inwards from the root endwall.

The turbine is fully instrumented with kiel rakes, wall static pressure tappings, and thermocouples from which both the individual stage and turbine efficiencies can be obtained. In addition the stage 2 diaphragm has wall static pressure tappings on the exit face to measure the pressure variation in the disc/diaphragm space. These were calibrated statically to \pm 5 mbar.

Two series of tests were carried out:

(1) Efficiency measurements were performed with a blade radius ratio of 1.11 and disc/diaphragm gaps of $4 \mathrm{~mm}$ and $2 \mathrm{~mm}$. Performance data and diaphragm face wall pressures were measured for dimensionless blade root speed $\left(\mathrm{u} / \mathrm{C}_{\mathrm{o}}\right)$ in the range 0.48 to 0.56 .

(2) Unsteady pressure measurements carried out with a blade radius ratio of 1.23 and a disc/diaphragm gap of $4 \mathrm{~mm}$. As shown in figs 2 and 3, fast response pressure transducers were incorporated in the disc/diaphragm space on stage 2 for these tests. The transducers were surface mounted Endevco piezoresistive $8515 \mathrm{C}-50$ (6.3mm diameter x $0.76 \mathrm{~mm}$ thick). The transducer outputs were recorded at a tape speed that would allow frequencies up to $20 \mathrm{kHz}$ to be postprocessed. The test data presented however were processed 'live' using an 'in house' LabVIEW based analysis tool. The transducers were calibrated statically to \pm 5 mbar, and had a resonance frequency of $320 \mathrm{kHz}$, two orders of magnitude greater than the blade passing frequency.

Measurements were recorded at conditions of either constant pressure level (1.57 bar at exit) and ratio (1.562) with varying speed $(2800-3500 \mathrm{rpm})$, or constant speed $(2800,3000,3300 \mathrm{rpm})$ with varying pressure level and ratio. Note however that for all unsteady tests the absolute pressure at the measurement plane was maintained at 1.88 bar.

\section{CFD MODELLING}

Meshes for the CFD models presented here were generated using the Gambit mesh generator versions 1.3 and 2.0 and the FLUENT solver versions 5.5 and 6.0 [11]. The basic solution domain and the coarser calculation mesh used for the initial axisymmetric study is illustrated in fig. 4. This included the cavity between the rotating and stationary discs and a section of the 
annulus. The 3D meshes covered a similar axisymmetric domain with either a $90^{\circ}$ or full $360^{\circ}$ sector being modeled. Neither the stationary vanes nor the rotating blades were included in any of the models, but some models included balance holes in the rotating disc. Where the balance holes are included, they are assumed to be 4 holes equally spaced around the circumference. This does not quite match the experiment in which the spacing is non-uniform.

Two meshes were used for the axisymmetric models. The coarser of these, shown in fig.4, has 6000 quadrilateral cells while the finer mesh had 24000 quadrilateral cells. The annulus inlet boundary conditions were set using the total pressure and flow angle profiles given by design calculations for the second stage vane exit conditions. The static pressure was specified at the hub for the annulus outlet with the radial pressure distribution being given by the radial equilibrium condition. In the rig, flow entered the disc cavity at the inner radius through a labyrinth seal. The geometry of this region was not modeled exactly, but flow was introduced using a specified mass flow condition with zero swirl velocity at the cavity inner radius. When the balance holes were not modeled the specified seal flow was reduced so that the net flow from the cavity into the annulus would match the value estimated for the rig. When balance hole flow was modeled, the pressure was specified at the hole exit and this was adjusted to give the required mass flow. Unless otherwise stated no-slip conditions were applied at all solid boundaries. Compressible flow assumptions and standard relations for the properties of air were used in the axisymmetric models.

Five different meshes were used in the $3 \mathrm{D}$ studies and these are summarized below. These include $90^{\circ}$ and $360^{\circ}$ sector models. The $90^{\circ}$ models assume circumferential periodicity whereas, no such assumption is required with the full $360^{\circ}$.

Mesh 1: An axisymmetric mesh for the $4 \mathrm{~mm}$ axial gap on a $90^{\circ}$ sector with 380000 hexahedral cells, as shown in fig. 5 . The mesh on each circumferential plane is identical, being obtained by rotation about the axis of revolution. There are no balance holes.

Mesh 2: Similar to mesh 1, for the $4 \mathrm{~mm}$ axial gap, but for a full $360^{\circ}$. This mesh is axisymmetric, has 1190000 hexahedral cells, and does not include balance holes.

Mesh 3: A $90^{\circ}$ sector, for the $4 \mathrm{~mm}$ axial gap, containing 1 of the 4 balance holes. The mesh has 660000 hexahedral cells and is shown in fig. 6.

Mesh 4: Similar to mesh 3, for the $4 \mathrm{~mm}$ gap, but for a full $360^{\circ}$. This mesh, has 2450000 hexahedral cells, and includes 4 balance holes.

Mesh 5: This is a $360^{\circ}$ axisymmetric mesh for the $2 \mathrm{~mm}$ axial gap having 1420000 hexahedral cells and not including any balance holes.

Boundary conditions applied for the 3D models are as described above for the axisymmetric case, with rotational periodicity assumed for the $90^{\circ}$ models. Compressibility was neglected for the $3 \mathrm{D}$ cases, with density assumed constant. This involved little 


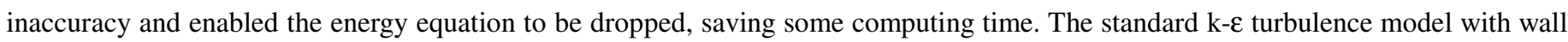
functions was used throughout. The three momentum balance equations, the continuity equation, and the turbulence model equations are solved using the SIMPLE pressure correction algorithm. Second order upwind spatial differencing was employed with a second order pressure correction equation. These methods have been used successfully for other rotating disc cavity and turbomachinery flows. However for the present problem, even when the geometry and boundary conditions were axisymmetric, attempts to find steady solutions produced 3D flow fields, with doubtful convergence. This led to all 3D models being solved in a time accurate fashion. This was done in the rotating frame of reference using implicit, second order time stepping. The time step used was $1.2 \times 10^{-4} \mathrm{~s}$ corresponding to 0.0064 of a disc revolution, and the simulation was continued for about $0.5 \mathrm{~s}$ (or about 26 disc revolutions). Steady 'solutions' were used as starting conditions for the unsteady calculations. For both 2D and 3D cases stable steady solutions were obtained by gradually increasing the rotor speed and the annulus inlet swirl to the desired values. Near-wall values were checked for all cases to ensure that the mesh was appropriate for application of the wall functions. Values of $\mathrm{y}+$ in the cavity for the $4 \mathrm{~mm}$ gap solutions were the range 10 to 300 . For the $2 \mathrm{~mm}$ gap solution the peak value was 500 .

\section{RESULTS}

CFD results were produced for a design point condition for which the rotational Reynolds number $\left(\operatorname{Re}_{\phi}=\rho \Omega \mathrm{r}_{\mathrm{o}}{ }^{2} / \mu\right)$ is $6.0 \times 10^{6}$, the mean annulus inlet Mach number and angle to the axial direction are 0.56 and $78^{\circ}$, with the mean inlet swirl equal to $1.83 \Omega r_{0}$. The seal flow feeding the cavity and the balance hole flow are $1.09 \%$ and $0.73 \%$ of the main annulus flow, respectively. This gives a net flow from the cavity into the annulus of $0.36 \%$ of the annulus flow, corresponding to a non-dimensional mass flow rate $\left(\mathrm{C}_{\mathrm{w}}=\mathrm{m} / \mu_{\mathrm{o}}\right)$ of 5867. This net flow rate is about $10 \%$ of the flow entrainment expected for a disc rotating in a quiescent environment at this rotational Reynolds number. The mean radial velocity based on the net mass flow is about 0.03 times the mean axial velocity in the annulus for the $4 \mathrm{~mm}$ axial gap, and twice this value for the $2 \mathrm{~mm}$ gap. The CFD solutions at these conditions are presented and compared with the experimental data in the following subsections.

\subsection{Mean pressure distributions}

Fig. 7 shows the comparison of measurements from pressure tappings on the stationary disc with axisymmetric CFD solutions from the two meshes. Also shown, for reference, are the lines given by a forced vortex at disc speed and a forced vortex at half disc speed. These results are presented in the form of a pressure coefficient referenced to the pressure at the disc outer radius and non- 
dimensionalised using the inlet dynamic head in the CFD solution. Note that an error of \pm 5 mbar in the pressure measurements, would give an error in $\mathrm{C}_{\mathrm{p}}$ of about \pm 0.008 . For a rotor-stator disc cavity with no through flow a 'core’ tangential velocity of about $40 \%$ of disc speed is expected. The much higher swirl levels indicated by the pressure measurements for the $4 \mathrm{~mm}$ axial gap may be attributed to ingestion of the highly swirling annulus gas into the cavity. Measurements for the $2 \mathrm{~mm}$ gap are fairly close to the half speed vortex line, indicating considerably less ingestion for the smaller gap. The CFD solutions for the $4 \mathrm{~mm}$ gap clearly under predict the pressure gradient and, by inference, the degree of ingestion into the cavity. Results from the two CFD meshes are very close, showing that the discrepancy with experiment is not due to numerical truncation error

The streamline plots in fig. 8 confirm the lack of ingestion in the axisymmetric CFD results. As expected, the solution shows recirculation in the cavity due to the disc pumping effect. For a non-swirling annulus flow the pumping action might have been expected to draw flow into the cavity from the annulus, but this will be resisted by the highly swirling flow in this case. Further axisymmetric CFD solutions with the balance hole flow modeled by a slot exit in the rotating disc have shown that inclusion of the balance hole flow only affected the pressure gradient and flow structure in the inner part of the cavity. Insensitivity to the annulus hub boundary layer was also demonstrated, through a solution with the inlet hub boundary layer removed and the annulus hub wall upstream of the axial gap treated as a slip boundary.

Although not part of the present study, it is interesting to note the results of Chen [12], who did some earlier comparisons of CFD and measurements for this case. With a 3D, steady CFD model including one stationary vane and the disc cavity Chen also found that a CFD model of the $4 \mathrm{~mm}$ gap geometry gave a considerably lower cavity pressure gradient than shown by the measurements.

Time averaged pressure predictions from the 3D, unsteady CFD models are compared with the measurements in fig. 9. Checks on the numerical solutions showed that these time averages agreed with instantaneous circumferential averages. It is immediately apparent that these results for the $4 \mathrm{~mm}$ gap are much closer to the experimental data than the axisymmetric model. The $90^{\circ}$ and $360^{\circ}$ models without balance holes show very similar mean pressures although, as will be shown later, both these solutions show large scale unsteady effects but differ in detail. The $90^{\circ}$ model with balance holes shows the greatest overall pressure difference across the cavity, with the $360^{\circ}$ model for the same geometry giving the best agreement with experiment. The difference between these $90^{\circ}$ and $360^{\circ}$ models is attributed to unsteadiness of the flow through the balance holes. The assumption of periodicity in the $90^{\circ}$ model forces mass flow through all 4 holes to be synchronous. In the $360^{\circ}$ model the balance hole flow variations are out of phase, and this reduces the overall degree of unsteadiness. CFD results for the $2 \mathrm{~mm}$ gap are in fair agreement with measurements, showing that the CFD models have captured the trend of ingestion decreasing as the gap is reduced. 


\subsection{Unsteady flow structure}

Instantaneous pressure and radial velocity contours for the $4 \mathrm{~mm}$ gap, $360^{\circ}$ model with an axisymmetric mesh are shown in fig. 10 . These contours are on an axial plane $0.529 \mathrm{~mm}$ upstream of the rotating disc. The axisymmetric contours in the outer part of the domain show the uniformity of the main annulus flow. At the rim gap, alternate regions of cavity inflow and outflow occur around the circumference. Associated pressure asymmetries are also evident. When viewed as a time series in the rotating frame these flow patterns rotate slowly in the opposite direction to the disc rotation. Thus in the absolute frame the flow patterns rotate at slightly less than disc speed. These large scale structures are thought to arise from instability of the basic axisymmetric flow solution and are associated with interaction of the main annulus and cavity flows. They are concentrated in the region of the cavity where the axial gap is constant at $4 \mathrm{~mm}$.

Fig. 11(a) shows pressure contours on the same plane for the $4 \mathrm{~mm}$ gap, $90^{\circ}$ model with an axisymmetric mesh. The enforced periodicity has clearly modified the structure compared to the $360^{\circ}$ model, but the size of the structures are similar. As mentioned above, it appears that these differences in flow detail have little influence on the overall level of ingestion as the mean cavity pressure gradients given by the two solutions are very close. The pressure contours for the $360^{\circ}$ model with balance holes again (Fig. $11(\mathrm{~b})$ ) shows some modification of the flow details but qualitatively very similar structures.

Instantaneous radial velocity and pressure contours from the $2 \mathrm{~mm}$ gap model are shown in fig. 12 . These show reductions in length scale and strength of the unsteady, 3 dimensional effects compared to the $4 \mathrm{~mm}$ gap solutions. This may be associated with the reduced radial extent of the region of the cavity with minimum axial spacing as well as the change in gap. The reduction in the axial gap was expected to reduce ingestion and this effect appears to be captured by the model. Note also that the scale of the pressure asymmetries is now closer to that of the 50 vanes. For this case the pressure asymmetries due to the vanes might have as much effect on ingestion as the flow features shown in fig. 12.

\subsection{Unsteady pressures}

Results from the pressure transducer 'root 2' (the position of which is shown in fig. 3) are presented in figs 13 to 15 . These have been processed in order to expose the low frequency events predicted by the CFD. The signals were sampled at $2000 \mathrm{~Hz}$. Low pass filtering to $90 \%$ of the Nyquist frequency (i.e. $900 \mathrm{~Hz}$ ) was undertaken prior to sampling digitally, so the signals are not 
influenced by frequencies above this value (for example blade passing at $\sim 3.5 \mathrm{kHz}$ ). Unsteadiness in the 600 to $800 \mathrm{~Hz}$ range is apparent in these figures.

Fig. 14. Show results for a range of rotor speeds at constant pressure level and ratio. The frequencies only increase slightly with speed, and can be compared with the engine order traces that are just visible as diagonal straight lines. Considering these and other results indicates that the frequency is a stronger function of annulus swirl velocity than rotor speed. The phase angle between measurements at different annular positions confirms that the events are rotating rather than pulsating with an angular speed slightly below the rotor speed and 11-14 events around the annulus. The unsteadiness was also found to be much stronger at the blade root positions than the rotor hub position shown in fig. 3. The time-frequency plot in fig. 15 suggests a degree of randomness with instabilities giving rise to a series of events in the 600 to $800 \mathrm{~Hz}$ frequency range. There may be a different number of events around the annulus at different times. This would concur with some of the effects seen in animations of the CFD results.

Time traces of pressure from the CFD simulations for the $4 \mathrm{~mm}$ and $2 \mathrm{~mm}$ gap, $360^{\circ}$ geometries are shown in figs 16 and 17 . These are for a reference point on the stator disc, but rotating with the frame of reference, at a radius of $304 \mathrm{~mm}$. The $4 \mathrm{~mm}$ gap geometry of fig. 16, corresponds to the experimental conditions, and the level of pressure variation is similar to that shown in the measurements in fig. 13. The CFD results show some randomness (like the experiments), but the $2 \mathrm{~mm}$ gap CFD results are much closer to a periodic solution. The $4 \mathrm{~mm}$ gap, $90^{\circ}$ sector model without balance holes, which are not shown here, also displayed a more periodic nature.

Comparison of experimental and CFD frequencies requires conversion between rotating and stationary frames. The speed of rotation of the flow pattern was estimated from changes over a small time period. This was not straightforward as the flow features change with time and do not all appear to move at exactly the same speed. However, in all cases examined the flow pattern tended to move (in the absolute frame) at slightly less than rotor speed, with estimates of 90 to $97 \%$ of rotor speed being typical. This is broadly in agreement with experiment. However the observed flow patterns suggest fewer events around the circumference (say 6 to 12 ) than was deduced from the measurements. Combining these results it appears that the dominant frequencies given by the CFD in the stationary frame would be lower than the measured values (say about 400 to $600 \mathrm{~Hz}$ ). Fourier analysis of the CFD results to identify the circumferential modes and tracking of these with time would give a more detailed comparison. This was attempted in one case, and better agreement with measurements was obtained, but further work would be needed to confirm this.

\section{CONCLUSIONS}


A combined CFD and experimental study has identified previously unknown unsteady flow features associated with interaction of rim seal and main annulus flows in an axial gas turbine. These unsteady features are clearly independent of blade passing events that have been shown in earlier studies to affect ingestion into the cavity in other geometries. The magnitude of the pressure perturbations is of order $10 \%$ of the dynamic head for the external flow, consistent with the disturbances arising from flow interaction as the rim seal and main gas path flows meet. The CFD models show that this is an incompressible flow phenomenon. The geometry considered here has a simple axial gap between rotor and stator discs with uniform cavity width at outer radii. The length scale for the disturbances may be linked to the radial extent of the uniform width region. As the gap width was reduced, keeping the same net rim seal flow, the ingestion was suppressed and the unsteadiness weakened. Although not demonstrated, it is expected that as the rim seal flow rate is increased, or the gap reduced further, the unsteadiness will be suppressed. The CFD results were obtained in advance of the unsteady pressure measurements, and so represent true predictions to a large extent. Excellent qualitative and some good quantitative agreement with measurement has been found. Time-averaged results for $90^{\circ}$ sector models show largely good agreement with those for $360^{\circ}$ degree models, although the details of the unsteady flow differ. An exception to this was the $90^{\circ}$ model for the 4 balance holes configuration. In this case the periodic condition forced all balance hole flows to be equal at any instant, and the overall level of unsteadiness was greater than in the $360^{\circ}$ model. The mesh sizes selected for the $3 \mathrm{D}$ calculations were guided by numerical tests for axisymmetric flow, but some uncertainty as to numerical accuracy remains. Turbulence model inaccuracies may also be significant.

While identification of these unsteady effects is regarded as a significant advance in our understanding of this area, there is clearly more work that could be done. Use and development of CFD for design and research will no doubt continue to expand in this area, and so will use of fast response instrumentation. The present authors are currently investigating the particular question as to how the identified effects influence turbine efficiency.

\section{ACKNOWLEDGMENTS}

The assistance of Dr Nick Hills with computing issues is much appreciated. The authors also gratefully acknowledge useful discussions with many colleagues. In particular Dr Jian-Xin Chen gave help with mesh generation and access to his initial CFD study, and Dr David Bell, Mr Tim Rice and Dr Peter Walker have given helpful input and advice. 


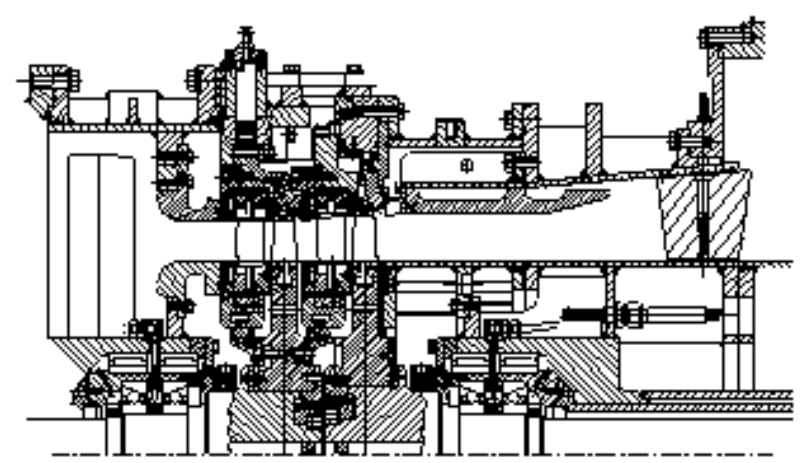

Fig. 1. Schematic of the 2 stage turbine rig.

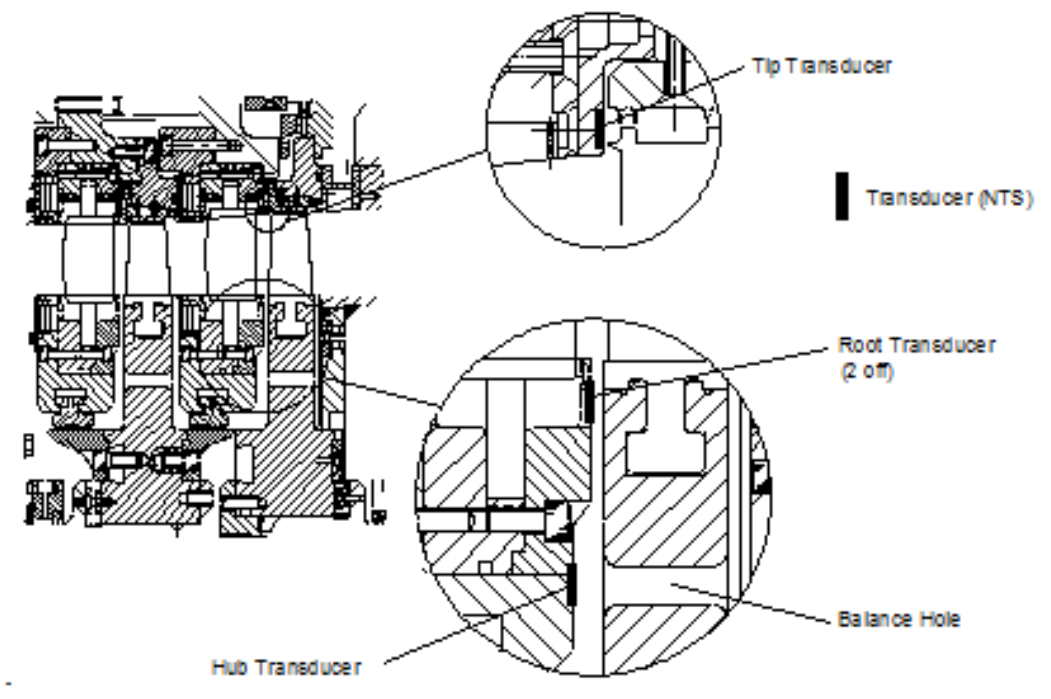

Fig. 2. Detail of the stage 2 disc/diaphragm space. 


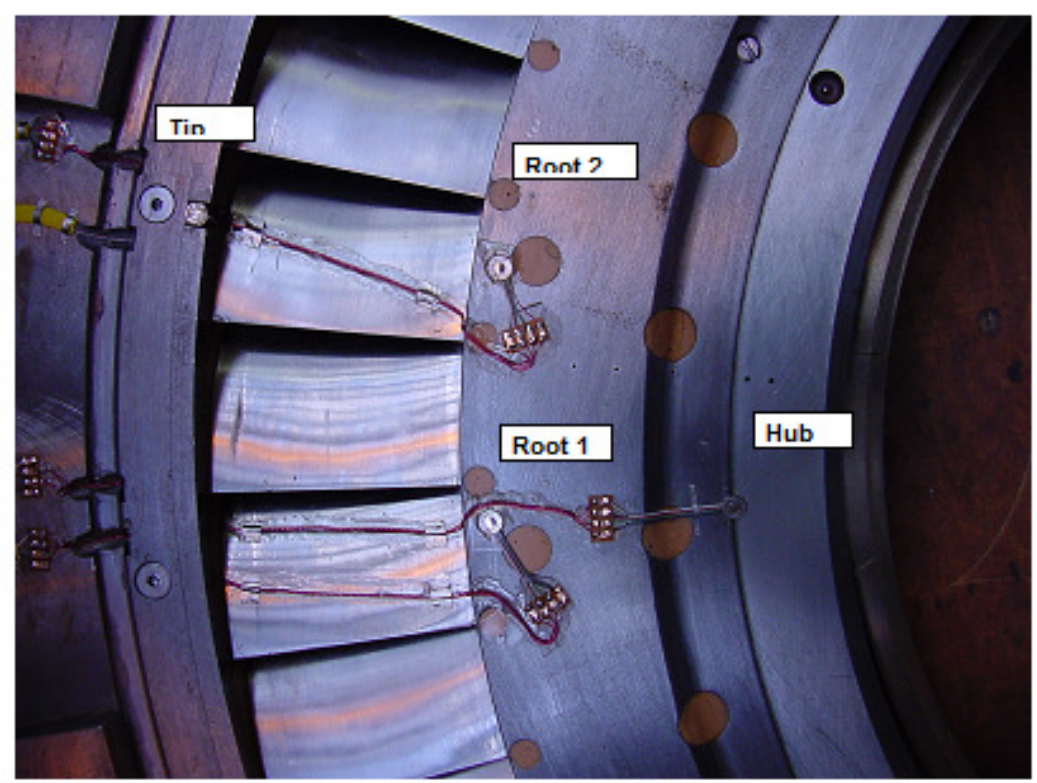

Fig. 3. Pressure transducer installation.

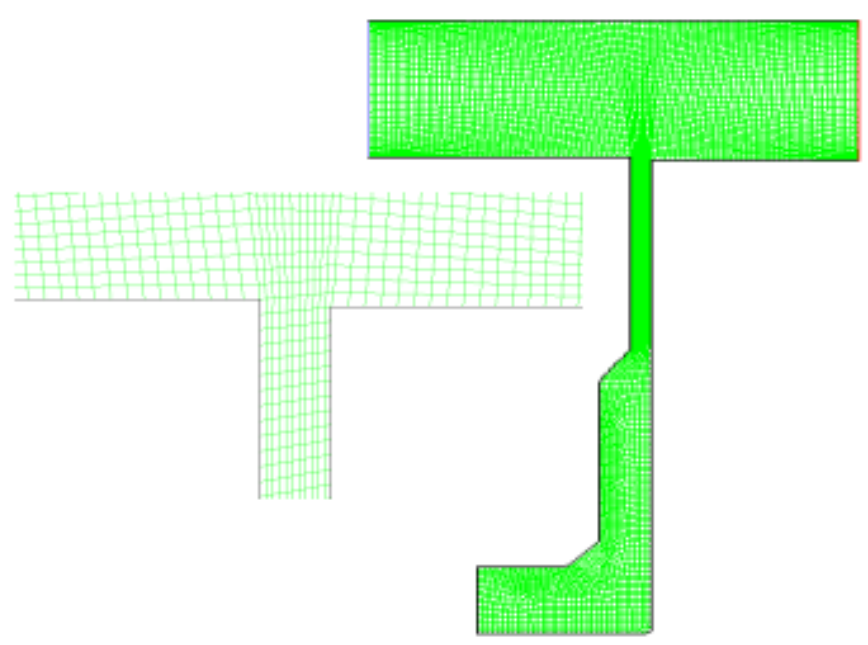

Fig. 4. Coarse mesh for the axisymmetric study. 


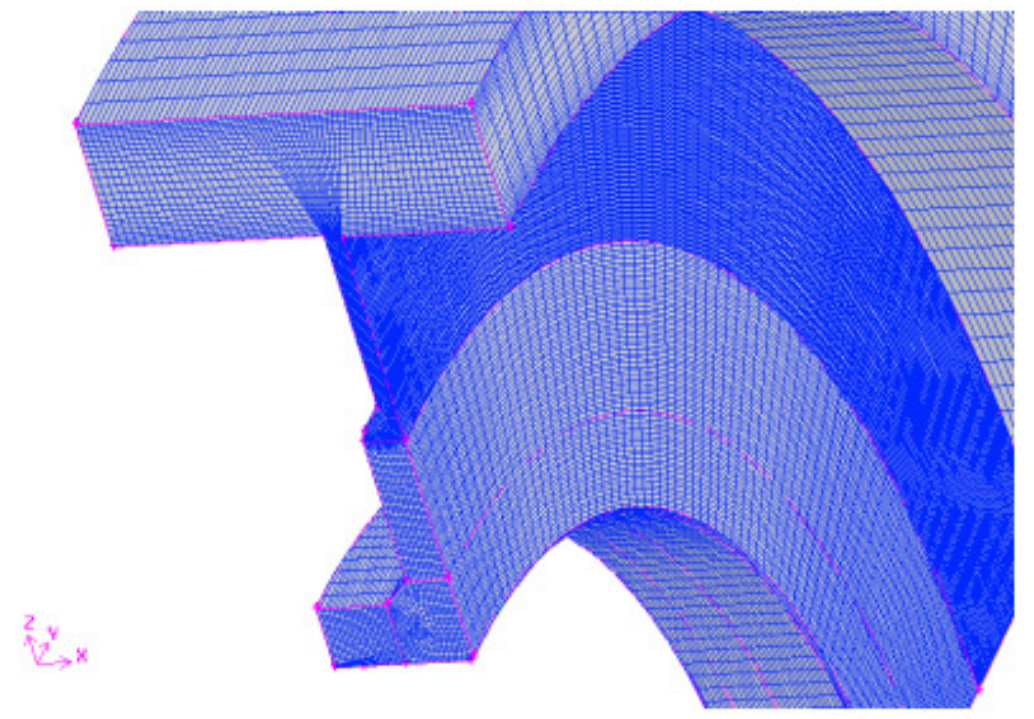

Fig. 5. Mesh 1: $4 \mathrm{~mm}$ axial gap on a $90^{\circ}$ sector.

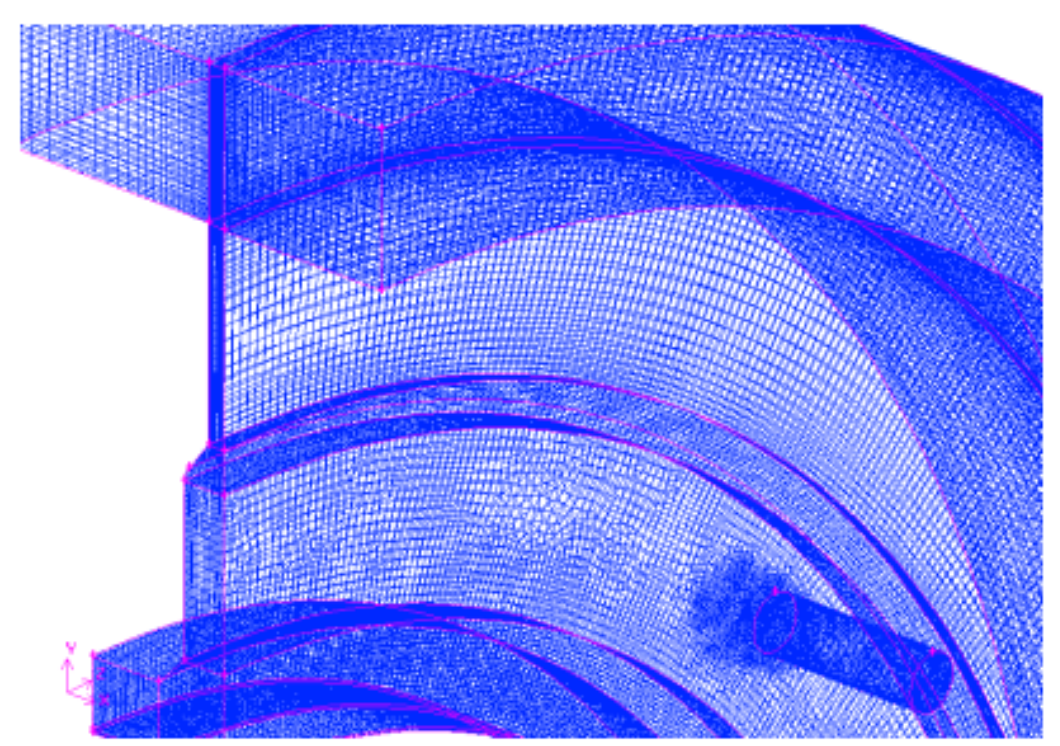

Fig. 6. Mesh 3: $4 \mathrm{~mm}$ axial gap with balance holes on a $90^{\circ}$ sector. 


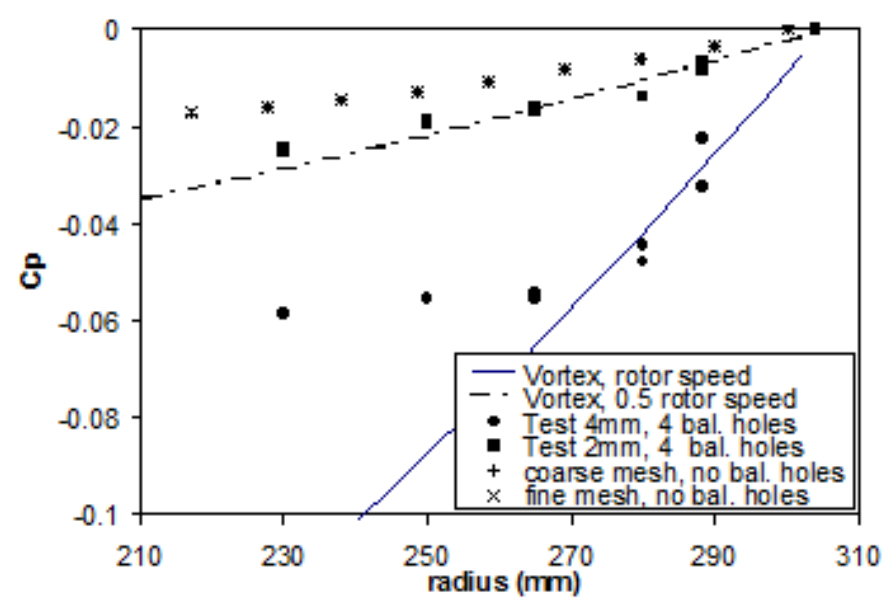

Fig. 7. Axisymmetric CFD results and measurements for the cavity pressure.

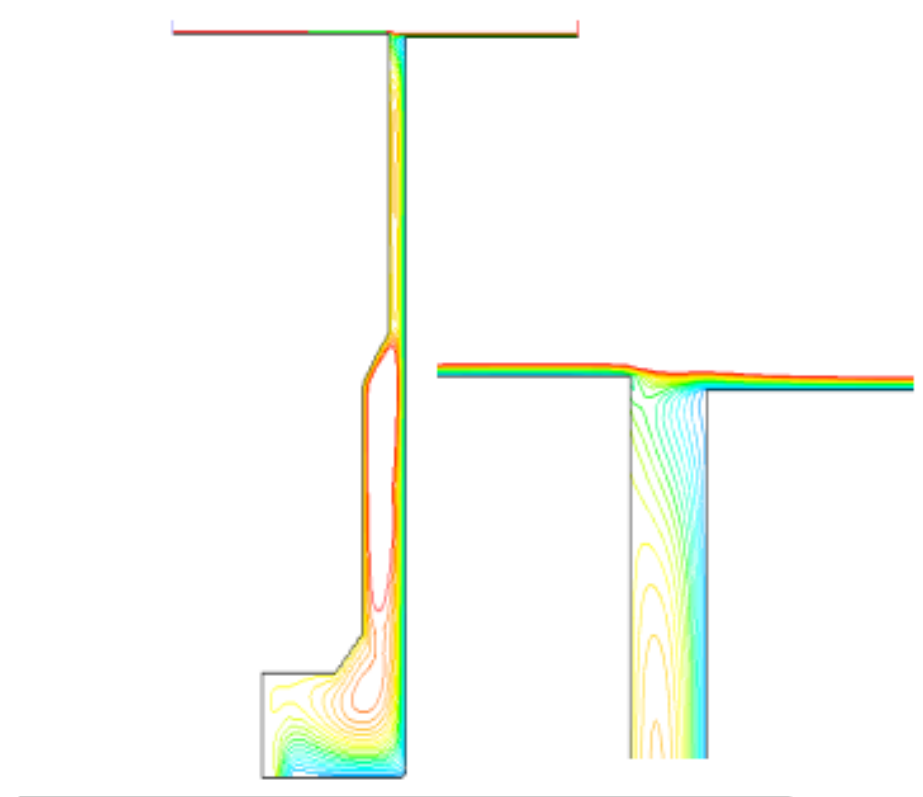

Fig. 8. Axisymmetric CFD flow streamlines for the fine mesh without balance hole flow. 


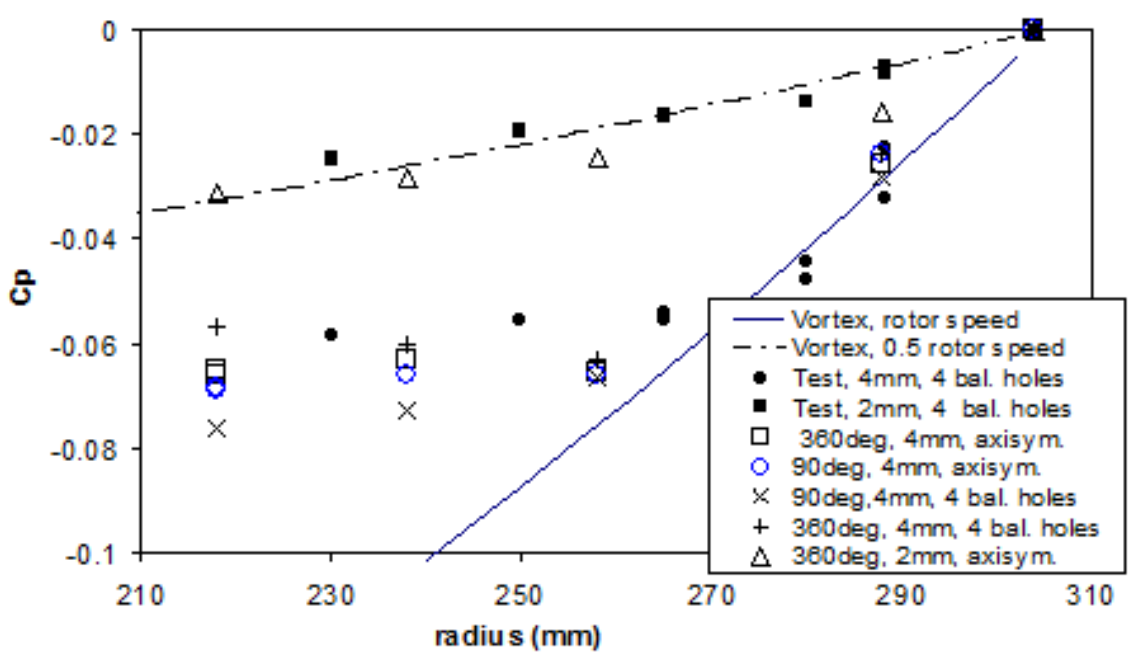

Fig. 9. 3D, unsteady CFD results and measurements for the mean cavity pressure. 

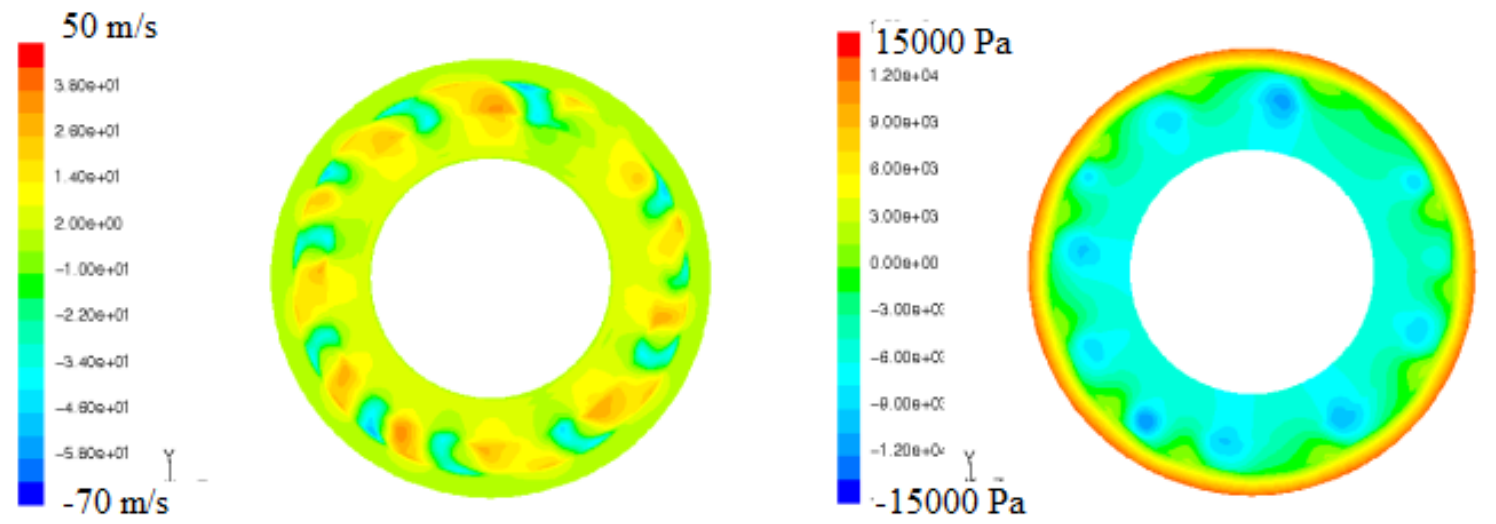

Fig. 10. Instantaneous radial velocities and pressure for the $4 \mathrm{~mm}$ gap, axisymmetric mesh, $360^{\circ}$ model.
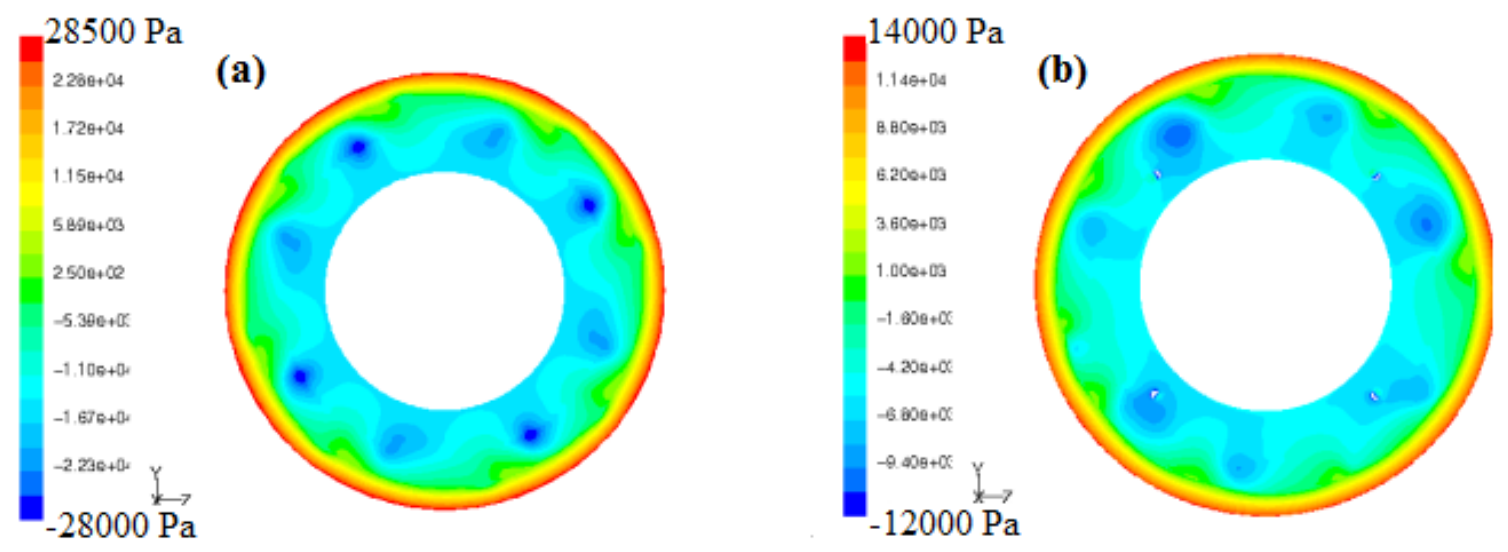

Fig. 11. Instantaneous pressure for (a) the $4 \mathrm{~mm}$ gap, axisymmetric mesh, $90^{\circ}$ model, and (b) the $4 \mathrm{~mm}$ gap, 4 balance holes, $360^{\circ}$ model.
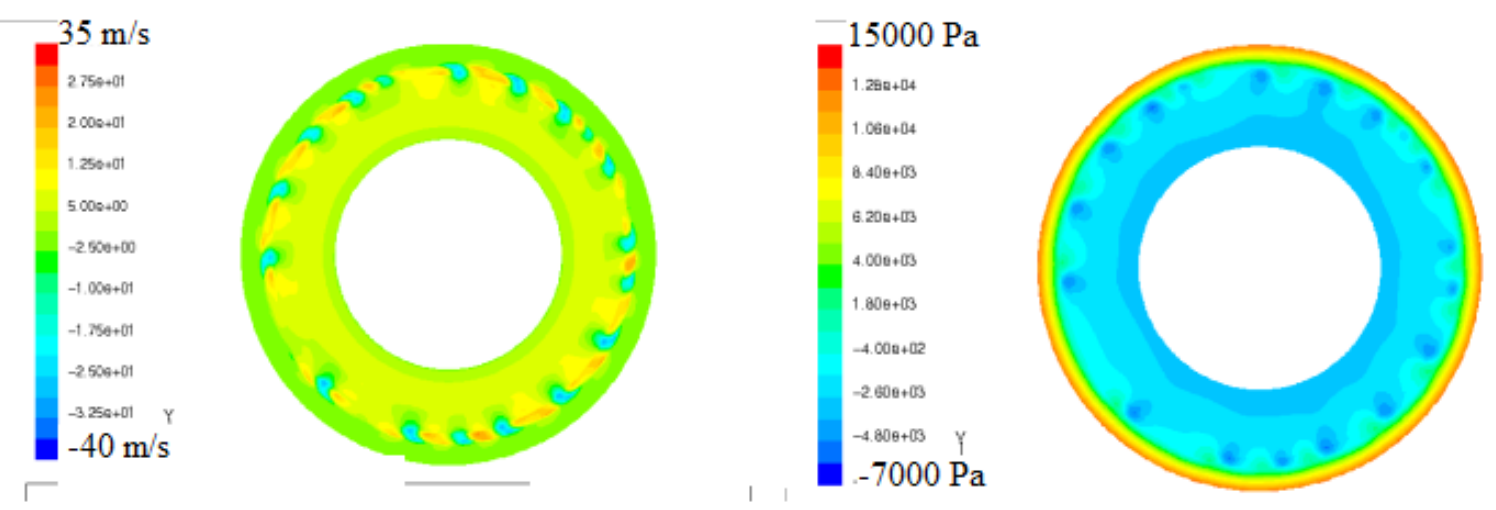

Fig. 12. Instantaneous radial velocities and pressure for the $2 \mathrm{~mm}$ gap, axisymmetric mesh, $360^{\circ}$ model. 


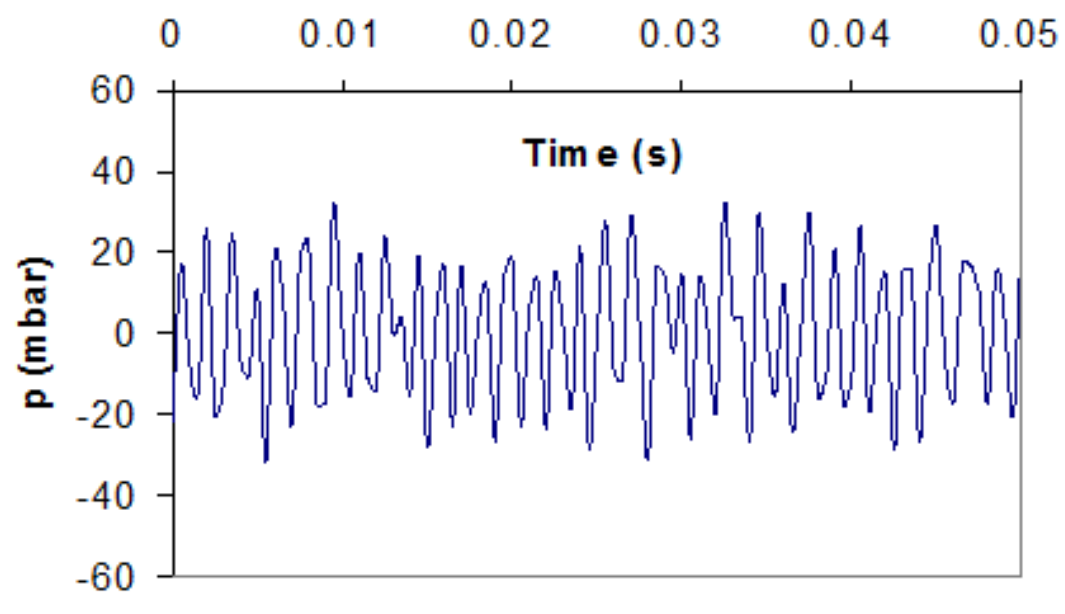

Fig. 13. Typical time trace for the filtered signal from the 'root 2' transducer.

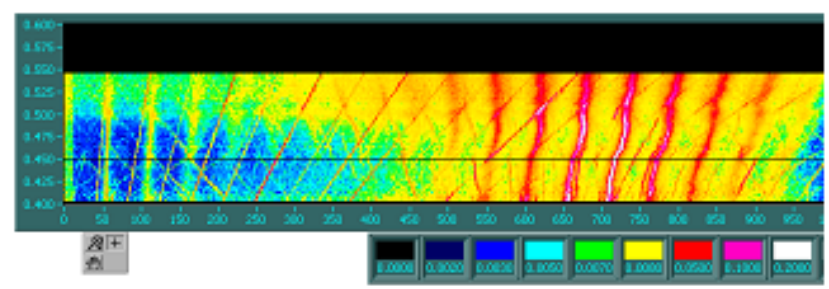

$\mathrm{X}$-Axis: Frequency, $\mathrm{Y}$-Axis: rotor $\mathrm{u} / \mathrm{C}_{\mathrm{o}}$, Intensity: $\mathrm{p}(\mathrm{rms}) / \mathrm{pAbs}^{*} 100 \%$

Fig. 14. Fourier analysis of results from 'root 2' transducer from a run with varying rotor speed. 


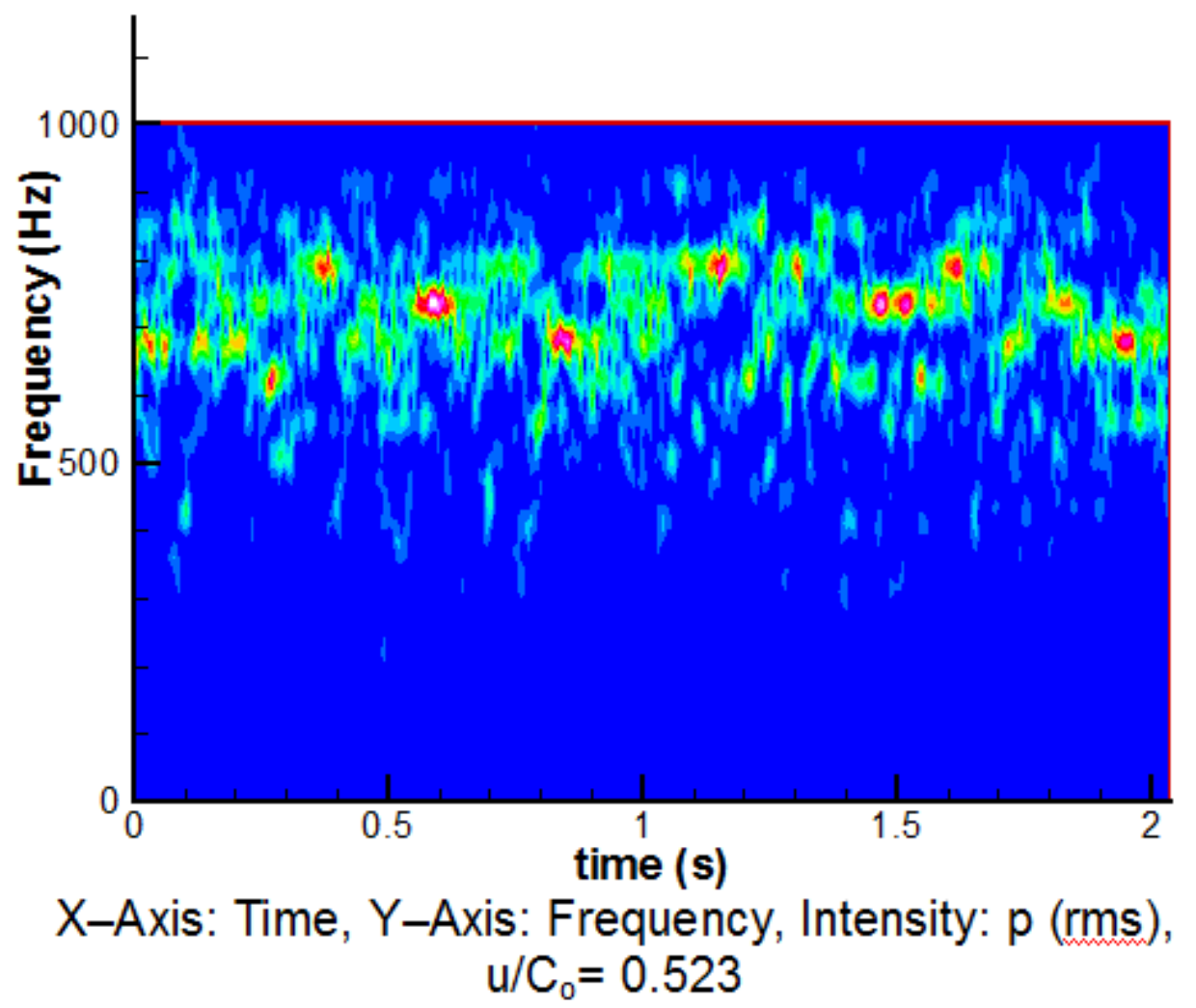

Fig. 15. Variation of intensity of frequency with time for the 'root 2' transducer. 


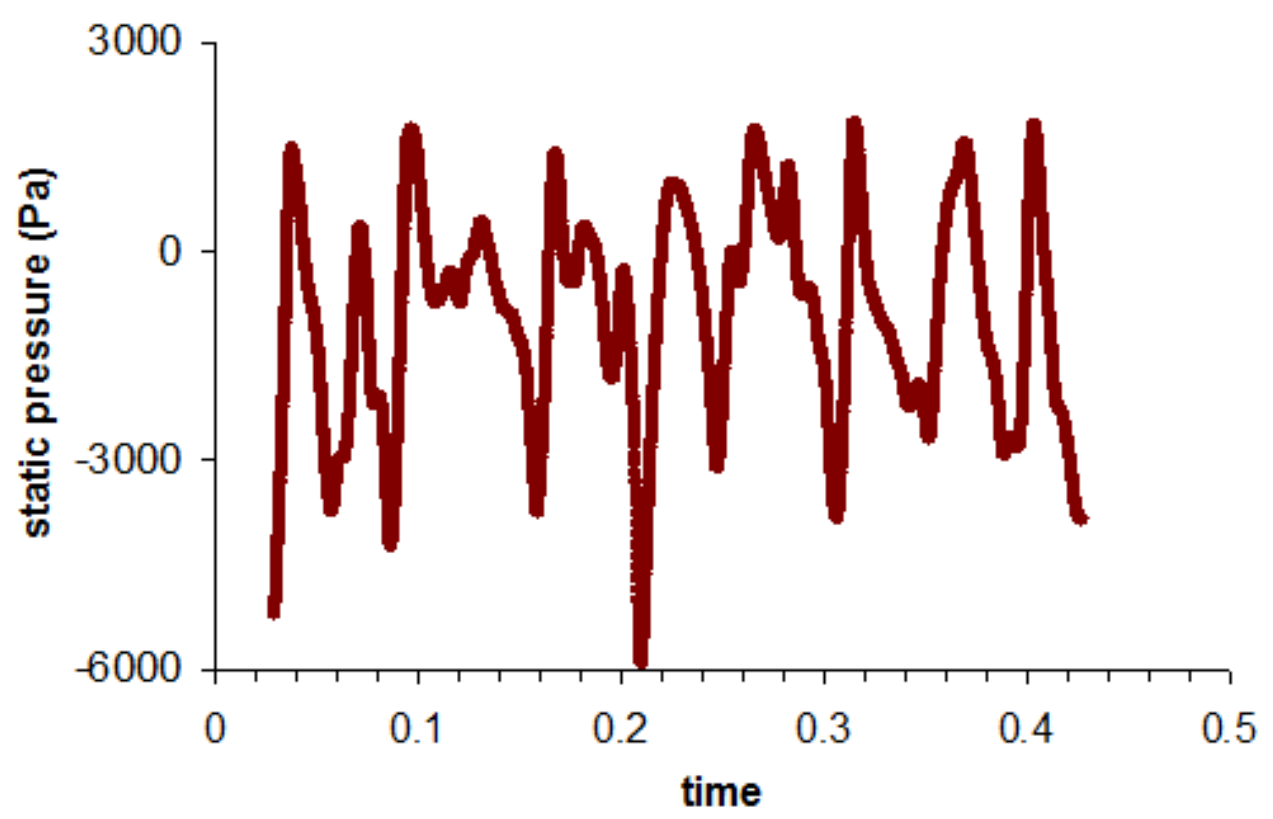

Fig. 16. Typical static pressure history (in the rotating frame) for the $360^{\circ}, 4 \mathrm{~mm}$ gap model without balance holes.

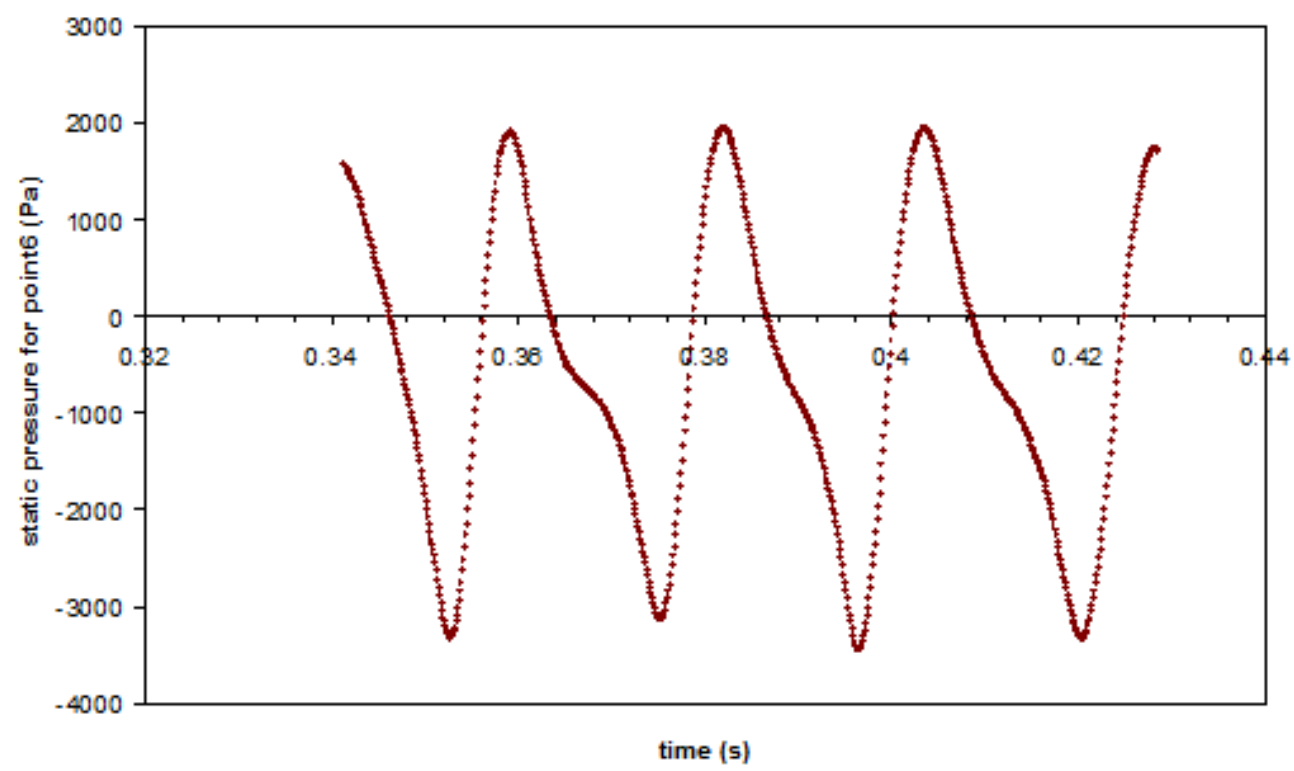

Fig. 17. Typical static pressure history (in the rotating frame) for the $360^{\circ}, 2 \mathrm{~mm}$ gap model without balance holes. 


\section{REFERENCES}

[1] Bayley, F.J. and Owen, J.M., 1970. The fluid dynamics of a shrouded disk system with a radial outflow of coolant. ASME J. Engng Power, vol. 92, pp. 335-341.

[2] Campbell, D. A., 1978. Gas turbine disc sealing system design. Proc. AGARD conf. On Seal technology in Gas Turbine Engines, AGARD-CP-237.

[3] Bohn, D., Rudzinsky, B., Surken, N. and Gartner, W., 2000, "Experimental and numerical investigation of the influence of rotor blades on hot gas ingestion into the upstream cavity of an axial turbine stage," ASME paper 2000-GT-284.

[4] Roy, R.P., Xu, G., Feng, J. and Kang, S., 2001, Pressure field and main stream gas ingestion in a rotor stator disc cavity. ASME Turbo Expo paper 2001-GT-564.

[5] Hills, N.J., Chew, J.W. and Turner, A.B., 2001, "Computational and mathematical modelling of turbine rim seal ingestion," ASME Turbo Expo paper 2001-GT-204 (Also ASME J. Turbomachinery, 2002,vol. 124, pp 306-315).

[6] Gentilhomme, O., Hills, N.J., Chew, J.W. and Turner, A.B., 2002. Measurement and analysis of ingestion through a turbine rim seal. ASME Turbo Expo paper GT-2002- 30481.

[7] Smout, P.D., Chew, J.W. and Childs, P.R.N., 2002. ICAS-GT: A European collaborative programme on internal cooling air systems for gas turbines. ASME Turbo Expo paper GT-2002- 30479.

[8] Bohn, D., Decker, A. and Wolff, M., 2003. Influence of sealing air mass flow on the unsteady hot gas ingestion in the upstream cavity of a 1.5 stage turbine. Proposed for ASME Turbo Expo.

[9]Autef, V. 2002. Computation of disc cavity rim sealing, University of Surrey/Institut National Polytechnique de Grenoble project report.

[10]Chew, J.W., Hills, N.J., Hornsby, C. and Young, C., 2003. Recent developments in application of CFD to turbomachinery internal air systems. Submitted to the 5th European Turbomachinery Conf., Prague. March 2003.

[11]Fluent Inc. http:Ilwww.fluent.com

[12] Chen, J-X., 2001. Alstom internal report, Whetstone, U.K. 